\title{
Unveiling Extreme Photoreduction Potentials of Donor-Acceptor Cy- anoarenes to Access Aryl Radicals from Aryl Chlorides
}

\author{
Jinhui Xu, Jilei Cao, Xiangyang Wu, Han Wang, Xiaona Yang, Xinxin Tang, Ren Wei Toh, Rong Zhou*, \\ Edwin K. L. Yeow, Jie $\mathrm{Wu}^{*}$
}

Supporting Information Placeholder

\begin{abstract}
Since the seminal work of Zhang in 2016, donor-acceptor cyanoarene-based fluorophores, such as 1,2,3,5-tetrakis(carbazol-9-yl)-4,6-dicyanobenzene (4CzIPN), have been widely applied in photoredox catalysis, and used as excellent metal-free alternatives to noble metal Ir- and Ru-based photocatalysts. However, all the reported photoredox reactions involving this chromophore family are based on harnessing the energy from a single visible light photon, with a limited range of redox potentials from -1.92 $\mathrm{V}$ to $+1.79 \mathrm{~V}$. Here, we document the unprecedented discovery that this family of fluorophores can undergo consecutive photoinduced electron transfer (ConPET) to achieve very high reduction potentials. One of the newly synthesized catalysts, 2,4,5-tri(9H-carbazol9-yl)-6-(ethyl(phenyl)amino)isophthalonitrile (3CzEPAIPN), possesses a long-lived (12.95 ns) excited radical anion form, $3 \mathrm{CzEPAIPN}{ }^{-*}$, which can be used to activate reductively recalcitrant aryl chlorides (Ered $\approx-1.9$ to $-2.9 \mathrm{~V}$ ) under mild conditions. The resultant aryl radicals can be engaged in synthetically valuable aromatic C-B, C-P, and C-C bond formation to furnish arylboronates, arylphosphonium salts, arylphosphonates, and spirocyclic cyclohexadienes, respectively.
\end{abstract}

Aryl chlorides are versatile building blocks in organic synthesis, being widely utilized as electrophilic partners in nucleophilic aromatic substitutions, in transition metal catalysis, and as precursors to organometallic complexes. Compared to the corresponding bromides or iodides, aryl chlorides are more abundant (Figure 1A), are frequently present in natural products and pharmaceutical molecules, and are often lower in cost, more stable, serving as excellent functional group handles for selective coupling ${ }^{1}$ or late-stage derivatizations. ${ }^{2}$

Photoredox catalysis offers numerous opportunities for the convenient synthesis of active organic radical species through single electron transfer (SET), which is complementary to conventional two-electron processes. ${ }^{3-6}$ However, compared to aryl bromides and iodides, aryl chlorides in photoredox catalysis have been largely underexplored due to the high energetic barrier for $\mathrm{C}\left(\mathrm{sp}^{2}\right)-\mathrm{Cl}$ bond activation $(\mathrm{PhCl}$ at approximately 95 $\mathrm{kcal} / \mathrm{mol}) .7,8$ Upon absorption of a photon, the excited photoredox catalyst promotes the single-electron reduction of aryl halides. Subsequent C-X bond cleavage delivers carbon-centered radicals, which can participate in a plethora of transformations. The reaction scope is therefore restricted by the energy of a photon. Visible light (e.g., a $440 \mathrm{~nm}$ blue photon possesses an energy of $2.8 \mathrm{eV}$ ) can activate only aryl chlorides possessing a strong electron-withdrawing substituent, ${ }^{9}$ and activation of more challenging nonactivated aryl chlorides requires carcinogenic high-energy ultraviolet (UV) light (Figure 1A).10,11

Several strategies have been developed during the past several years to address these challenges and enable the activation of aryl chlorides under visible light irradiation and mild conditions, such as consecutive photoinduced electron transfer (ConPET), sensitization-initiated electron transfer (SenI-ET), electrophotocatalysis, and solvated electrons.7,12-14 Among these strategies, ConPET represents the most operationally simple and green method ${ }^{12}$ and does not require additives such as metal catalysts, ${ }^{15}$ additional photosensitizers, ${ }^{16}$ micelles, ${ }^{17}$ electrolytes, or specialized electrochemical equipment. ${ }^{18,19}$ The
ConPET strategy relies on a photocatalyst and a sacrificial electron donor that, upon visible light irradiation, results in a longlived radical anion, which can be excited by a second photon to induce a much more powerful reducing species. ${ }^{20}$ The König group pioneered this field by developing a perylene bisimide (PDI) photocatalyst whose photoreduced form can undergo consecutive absorption of a second photon to achieve a much stronger reducing species for aryl halide reduction. ${ }^{21}$ Subsequently, several other organic dyes, including rhodamine $6 \mathrm{G},{ }^{22}$ 9,10-dicyanoanthracene (DCA), ${ }^{23}$ anthraquinone, ${ }^{24}$ acridinium salts (Mes-Acr ${ }^{2},{ }^{25}$ and benzo[ghi]perylene (BPI), ${ }^{26}$ were disclosed to be competent ConPET catalysts for aryl halide reduction, Birch reduction, ${ }^{26}$ and sulfonamide cleavage. ${ }^{25}$ However, the majority of these ConPET catalysts are still effective only for electron-poor aryl chlorides, ${ }^{21-24}$ and efficient catalytic systems for reductively recalcitrant aryl chlorides are rare ${ }^{25}$ but highly desirable. ${ }^{27}$

Carbazoyl dicyanobenzenes were first reported as highly efficient thermally activated delayed fluorescence (TADF) emitters by Adachi and coworkers. ${ }^{28}$ Among these donor-acceptor fluorophores, 1,2,3,5-tetrakis(carbazol-9-yl)-4,6-dicyanobenzene (4CzIPN) has been widely applied as a versatile metal-free photoredox catalyst to replace $\mathrm{Ru}$ - and Ir-polypyridyl photocatalysts. ${ }^{29,30}$ Its photophysical and electrochemical properties can be fine tuned by structural modification of the electron donor or electron acceptor moieties of the cyanobenzene scaffold to expand the redox window $(-1.92 \mathrm{~V}$ to $+1.79 \mathrm{~V}) .{ }^{31}$ Upon absorption of a blue photon, the excited 4CzIPN can be quenched via single-electron reduction, resulting in the formation of the radical anion $4 \mathrm{CzIPN}^{\bullet-}$. In the established $4 \mathrm{CzIPN}$-based photoredox catalysis process, this radical anion species is typically oxidized to regenerate the parent catalyst to complete the catalytic cycle (Figure 1B, left cycle). We herein report an unprecedented discovery that donor-acceptor cyanoarenes can effectively undergo a ConPET process to achieve a very strongly 

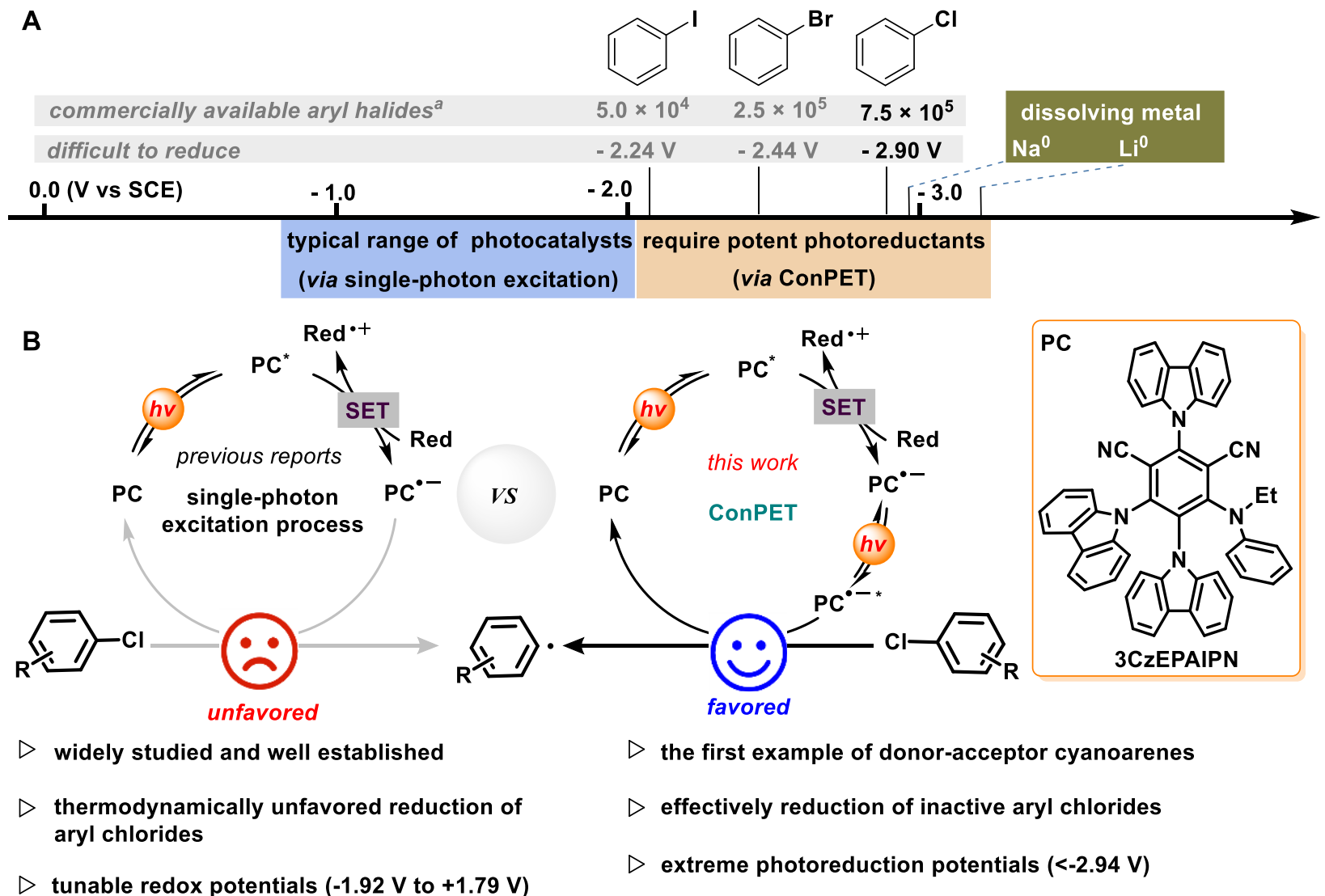

Figure 1. (A) Photocatalytic activation of aryl halides. (B) Disclosure of donor-acceptor cyanoarenes as efficient ConPET catalysts for aryl chloride activation. ${ }^{a}$ Commercial availability of different arene sources: SciFinder, accessed April 2nd, 2021. SCE $=$ saturated calomel electrode.

reducing photocatalytic system that can effectively reduce unactivated and electron-rich aryl chlorides (with reduction potentials, Ered, as low as $-2.94 \mathrm{~V}$ ) for aromatic C-B, C-P, and C-C bond formation (Figure 1B, right cycle). This strategy features advantages such as not requiring transition metals, being operationally simple, proceeding under mild conditions, and functioning with a wide range of aryl halides including complex pharmaceutical molecules for late-stage derivatization.

Our study was first inspired by the observation that the radical anion of 4CzIPN (4CzIPN ${ }^{-}$) generated from photoexcited 4CzIPN* and an electron donor $\mathrm{N}, \mathrm{N}$-diisopropylethylamine (DIPEA) is a long-lived radical anion species (stable for more than $3 \mathrm{~h}$ under argon in the dark; see details in the SI). This species can be further excited by $456 \mathrm{~nm}$ light to give a fluorescence band $\left(\lambda_{\max }^{e m}=448 \mathrm{~nm}\right)$ which is distinctly different from that of $4 \mathrm{CzIPN}^{*}\left(\lambda_{\max }^{e m}=545 \mathrm{~nm}\right.$, Figure S6). The reductive potential of $4 \mathrm{CzIPN}\left[\mathrm{E}_{1 / 2}\left(\mathrm{PC} / \mathrm{PC}^{\bullet-}\right)=-1.24 \mathrm{~V}\right.$ vs SCE$]$ is more negative than those of DCA, PDI, and Mes-Acr ${ }^{+}$and similar to that of $\mathrm{BPI}$, indicating that $4 \mathrm{CzIPN}^{{ }^{-*} *}$ is a powerful reducing species that can effectively reduce difficult aryl halides (Figure S8). ${ }^{25}$

We first attempted to develop a synthetically useful borylation reaction of chloroarenes ${ }^{32-34}$ based on the proposed ConPET process. Our initial optimization revealed that when 4CzIPN was used as a photocatalyst, with a catalytic amount of 4-cyanopyridine, sodium oxalate as an electron donor, and $\mathrm{K}_{3} \mathrm{PO}_{4}$ as a base, borylation of para-chlorotoluene 1 afforded the corresponding boronic ester 3 in $76 \%$ yield under $40 \mathrm{~W}$ blue LED irradiation (Table 1A, entry 2). 4CzIPN was not stable and decomposed during the reaction process, which may cause a lower reaction efficiency. We thus synthesized a group of new donor-acceptor dicyanobenzenes through a straightforward two-step procedure 29,35 in order to increase the stability of the photocatalyst during the ConPET process. Remarkably, an improved yield was obtained when using tri(9H-carbazol-9-yl)-6(methyl(phenyl)amino)isophthalonitrile (3CzMPAIPN) as the photocatalyst (entry 3), and further improvement to $95 \%$ isolated yield could be achieved by replacing the methyl substituent with an ethyl group (3CzEPAIPN, entry 1). Notably, none of the photocatalysts has a sufficiently strong reducing singlet excited state to reduce para-chlorotoluene $\mathbf{1}\left(\mathrm{E}_{\mathrm{red}}=-2.84 \mathrm{~V}\right)(\mathrm{Ta}$ ble 1B). When DIPEA was used as the electron donor instead of sodium oxalate, a moderate yield (52\%) was achieved due to competing dechlorinated byproduct formation (Table 1A, entry 4). Using sodium ascorbate as the electron donor afforded the same efficiency as that with sodium oxalate (entry 5); thus, inexpensive sodium oxalate was employed in the scope study. Eliminating the catalytic 4-cyanopyridine led to a substantially reduced yield (entry 6), indicating its important role for the stable boryl radical formation. ${ }^{36}$ The reaction became less efficient in the absence of $\mathrm{K}_{3} \mathrm{PO}_{4}$ (entry 7). Control experiments revealed the essential roles of both sodium ascorbate and photoirradiation as no product was detected in the absence of either of them (entries 8 and 9). Gram-scale synthesis was easily achieved by employing more intensive light irradiation and a longer reaction time (entry 10), demonstrating the synthetic practicality of this method.

With the optimized conditions in hand, we examined the scope of borylation of aryl chlorides through the ConPET process. We found that this protocol was effective with not only 
activated aryl chlorides (15-25) but also challenging nonactivated aryl chlorides (14) and electron-rich aryl chlorides (3-13) that possess very negative reduction potentials (up to $-2.94 \mathrm{~V}$, Table 2A). A number of functional groups were well tolerated, including ethers (8-11), phenols (12), sulfides (13), trifluoromethyl (24), and borates (25), as well as those potentially sensitive to strongly reducing conditions, such as aryl fluorides (16-19), nitriles (20-22), esters (23), indoles (26), and epoxides (27). Diborylation products $(\mathbf{2 5}, \mathbf{2 8}, \mathbf{2 9})$, which have found wide application in materials science, were obtained

Table 1. Optimization of Conditions for the Visible LightDriven Borylation of Aryl Chlorides

\begin{tabular}{|c|c|c|c|}
\hline & ( & $\begin{array}{l}\text { 3CzEPAIPN ( } 5 \text { mol\%), } 4-C N-P y(10 \text { mol\%) } \\
\mathrm{Na}_{2} \mathrm{C}_{2} \mathrm{O}_{4}\left(1.5 \text { equiv), } \mathrm{K}_{3} \mathrm{PO}_{4}(2.0 \text { equiv) }\right.\end{array}$ & \\
\hline 1 & 2 & MeCN (1 M), Blue LED $(2 \times 40 \mathrm{~W}, 456 \mathrm{~nm})$ & BPin \\
\hline $0.2 \mathrm{mmol}$ & 2.0 equiv & & \\
\hline Entry & Variation $\mathrm{t}$ & to standard conditions & Yield of $3(\%)^{a}$ \\
\hline 1 & none & & $96(95)^{c}$ \\
\hline 2 & 4CzIPN ins & stead of 3CzEPAIPN & 76 \\
\hline 3 & 3CzMPAIP & PN instead of 3CzEPAIPN & 85 \\
\hline 4 & DIPEA $(2.4$ & 4 equiv) instead of $\mathrm{Na}_{2} \mathrm{C}_{2} \mathrm{O}_{4}$ and $\mathrm{K}_{3} \mathrm{PO}_{4}$ & 52 \\
\hline 5 & L-AscNa & (1.5 equiv) instead of $\mathrm{Na}_{2} \mathrm{C}_{2} \mathrm{O}_{4}$ & 95 \\
\hline 6 & no $4-C N-P y$ & & 26 \\
\hline 7 & no $\mathrm{K}_{3} \mathrm{PO}_{4}$ & & 33 \\
\hline 8 & no $\mathrm{Na}_{2} \mathrm{C}_{2} \mathrm{O}$ & & 0 \\
\hline 9 & no light, or & rno 3CzEPAIPN & 0 \\
\hline $10^{b}$ & $6 \mathrm{mmol}$ of 1 & 1 instead of $0.2 \mathrm{mmol}$ & $79^{c}(1.03 \mathrm{~g})$ \\
\hline
\end{tabular}

B) Synthesis and characterization of new donor-acceptor dicyanobenzenes

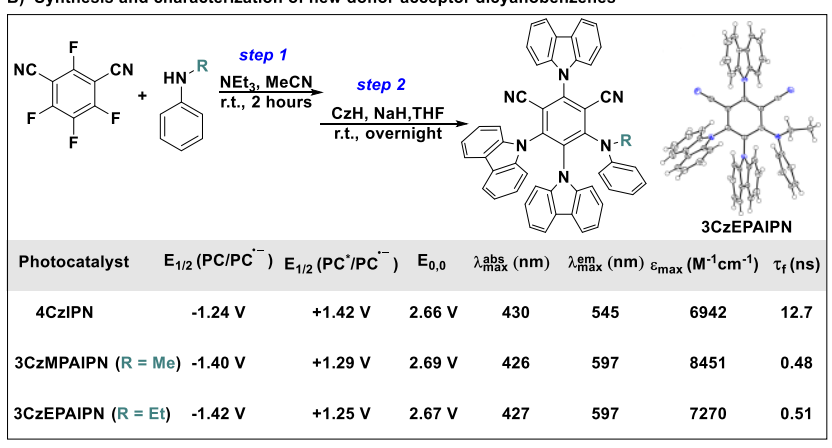

${ }^{a}$ Yields based on analysis of the ${ }^{1} \mathrm{H}$ NMR spectra of the crude product mixture using $\mathrm{CH}_{2} \mathrm{Br}_{2}$ as an internal standard. ${ }^{b}$ Blue LED ( 4 x $40 \mathrm{~W}, 456 \mathrm{~nm}$ ); the reaction was performed for $36 \mathrm{~h}^{c}$ Isolated yields. 4-CN-Py = 4-cyanopyridine; $\mathrm{L}-A s c N a_{2}=\mathrm{L}(+)$ ascorbic acid disodium salt; $\mathrm{E}_{0,0}=$ excited state energy; $\varepsilon_{\max }=$ molar extinction coefficient at the maximum absorption wavelength; $\tau_{\mathrm{f}}=$ lifetime of the excited state; $\mathrm{CzH}=$ carbazole.

good to excellent yields when dihaloarenes were employed, probably because the generated monoborylated chloride intermediates were more reducible than dihaloarenes. Aryl borates other than pinacolborate could be smoothly generated using the corresponding diboron esters (30-32, Table 2B). However, $\mathrm{B}_{2}(\mathrm{OH})_{4}$ was not compatible with our method due to its insolubility in acetonitrile (33).

A wide range of pharmaceutical compounds performed well under a slightly modified borylation protocol by using DIPEA as the electron donor, which possesses multiple Lewis basic atoms and heterocyclic moieties that can potentially coordinate and be problematic in transition metal catalysis (Table 2C).
Borylated derivatives of loratadine (34), meclizine (35), indomethacin (36), carbinoxamine (37), chloropyramine (38), cloperastine (39), chlorpromazine (40), and clofibrate (41) were generated in moderate to good yields. Some of the borylation products were isolated as organotrifluoroborate salts as a part of the workup procedure to assist product isolation.

The scope of this carbazoyl dicyanobenzene-promoted ConPET strategy could be extended to the formation of C-P bonds by employing phosphines or phosphites as radical trapping

Table 2. Scope of Visible Light-Induced Borylation of Aryl Chlorides $^{a}$

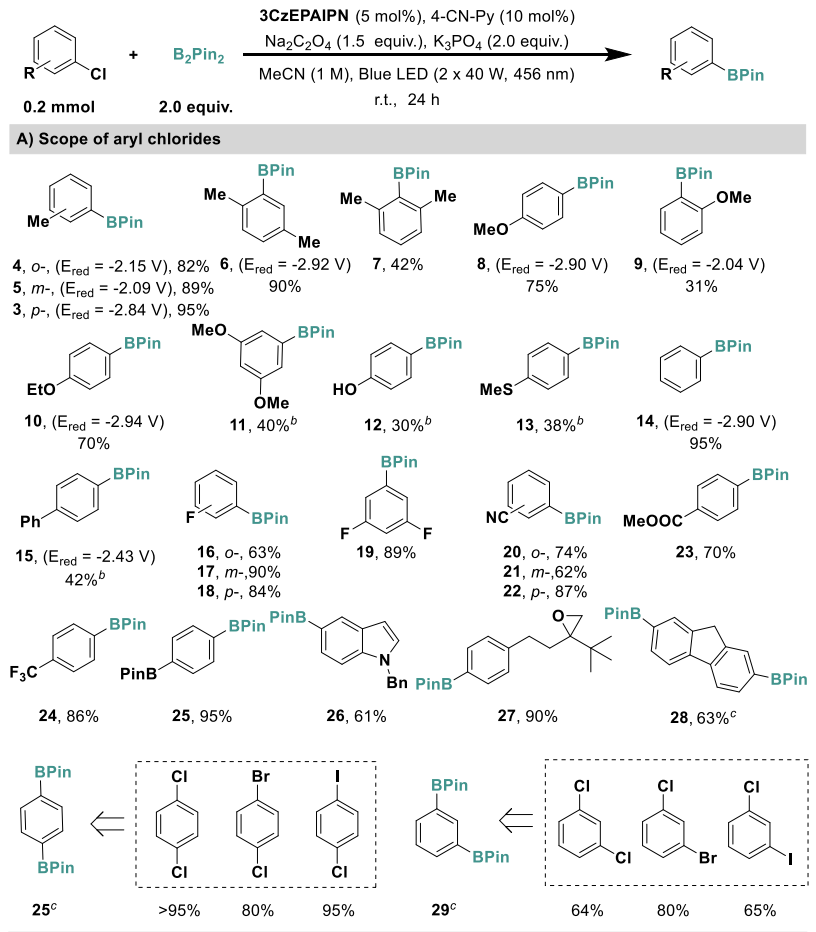

B) Scope of boronate esters
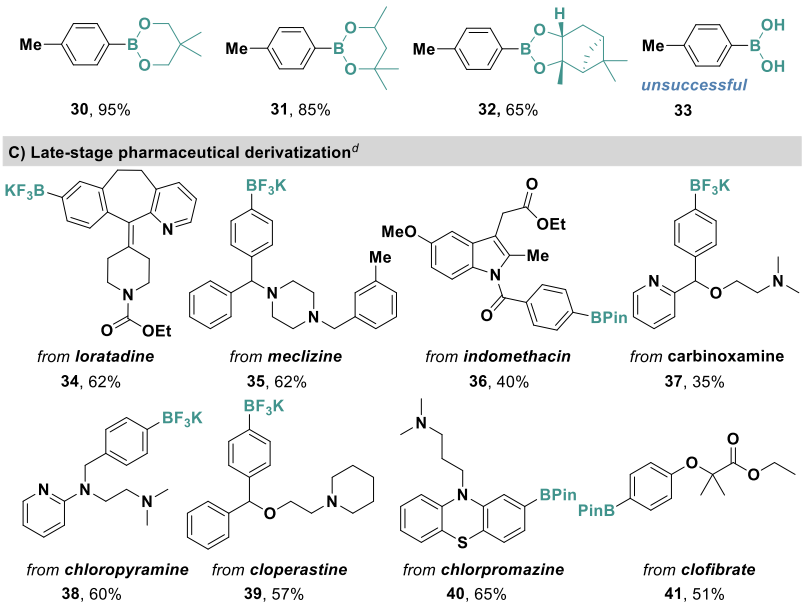

${ }^{a}$ Reaction conditions: aryl chloride (0.2 mmol), $\mathrm{B}_{2} \mathrm{Pin}_{2}(2.0$ equiv), 4-CN-Py (10 mol\%), $\mathrm{Na}_{2} \mathrm{C}_{2} \mathrm{O}_{4}$ (1.5 equiv), $\mathrm{K}_{3} \mathrm{PO}_{4}(2.0$ equiv), and 3CzEPAIPN ( $5 \mathrm{~mol} \%$ ) in MeCN (1 M) under irradiation with Kessil light ( $456 \mathrm{~nm}, 40 \mathrm{~W}$ ) at r.t. for $24 \mathrm{~h}$ under $\mathrm{N}_{2}$. Isolated yields. ${ }^{b}$ The reaction was performed for $36 \mathrm{~h} .{ }^{c} \mathrm{Only}$ diborylation products were obtained. $d$ Reaction conditions were slightly modified; see details in SI. 


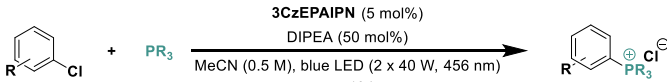

$$
\begin{aligned}
& \mathbf{R} \mathbf{2} \text { mmol } 2 \text { equiv } \mathrm{Cl} \text { r.t., } 48 \mathrm{~h}
\end{aligned}
$$

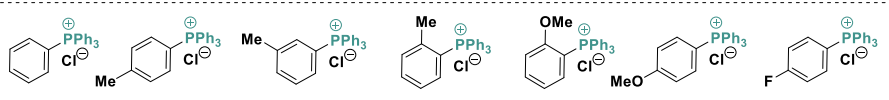

$\begin{array}{lllllll}42,87 \% & 43,75 \% & 44,90 \% & 45,60 \% & 46,80 \% & 47,40 \%{ }^{a} & 48,80 \%\end{array}$

(2),87\%

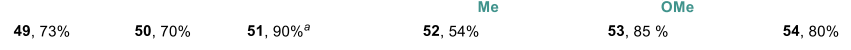

B) Phosphorylation of aryl chlorides with phosphites

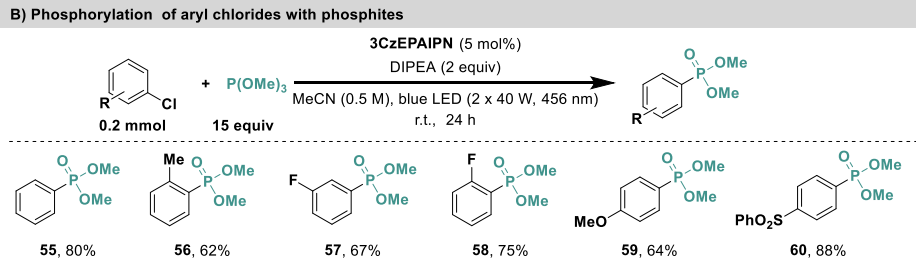

C) Intramolecular dearomative hydroarylation

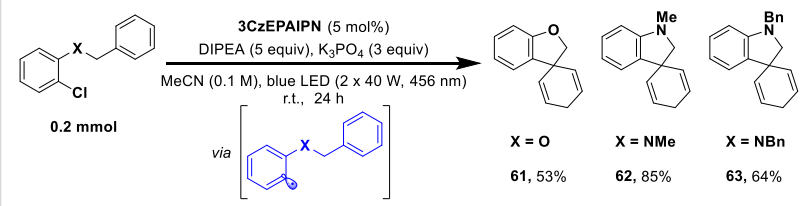

D) Hydrodefluorination of trifluoromethylarenes

$$
\text { 0.2 mmol }
$$

E) Scale-up by continuous-flow synthesis

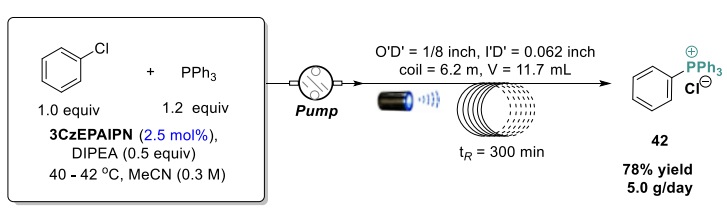

Figure 2. Various transformations promoted by the donor-acceptor cyanoarene-catalyzed reductive ConPET process. aUsing 4CzIPN instead of 3CzEPAIPN.

A

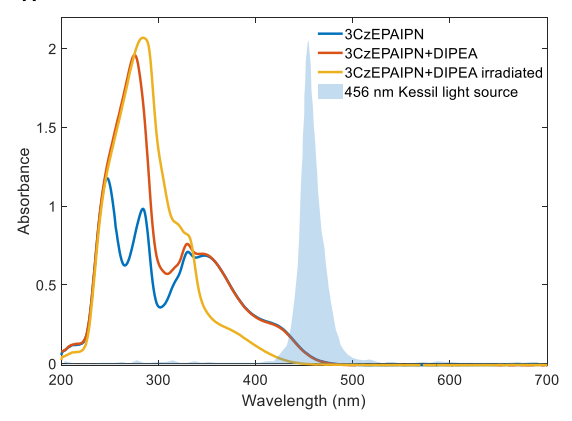

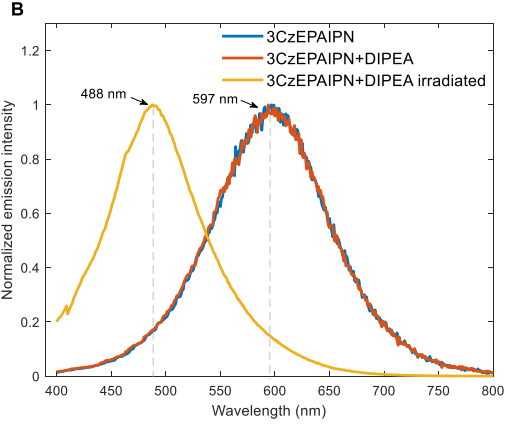
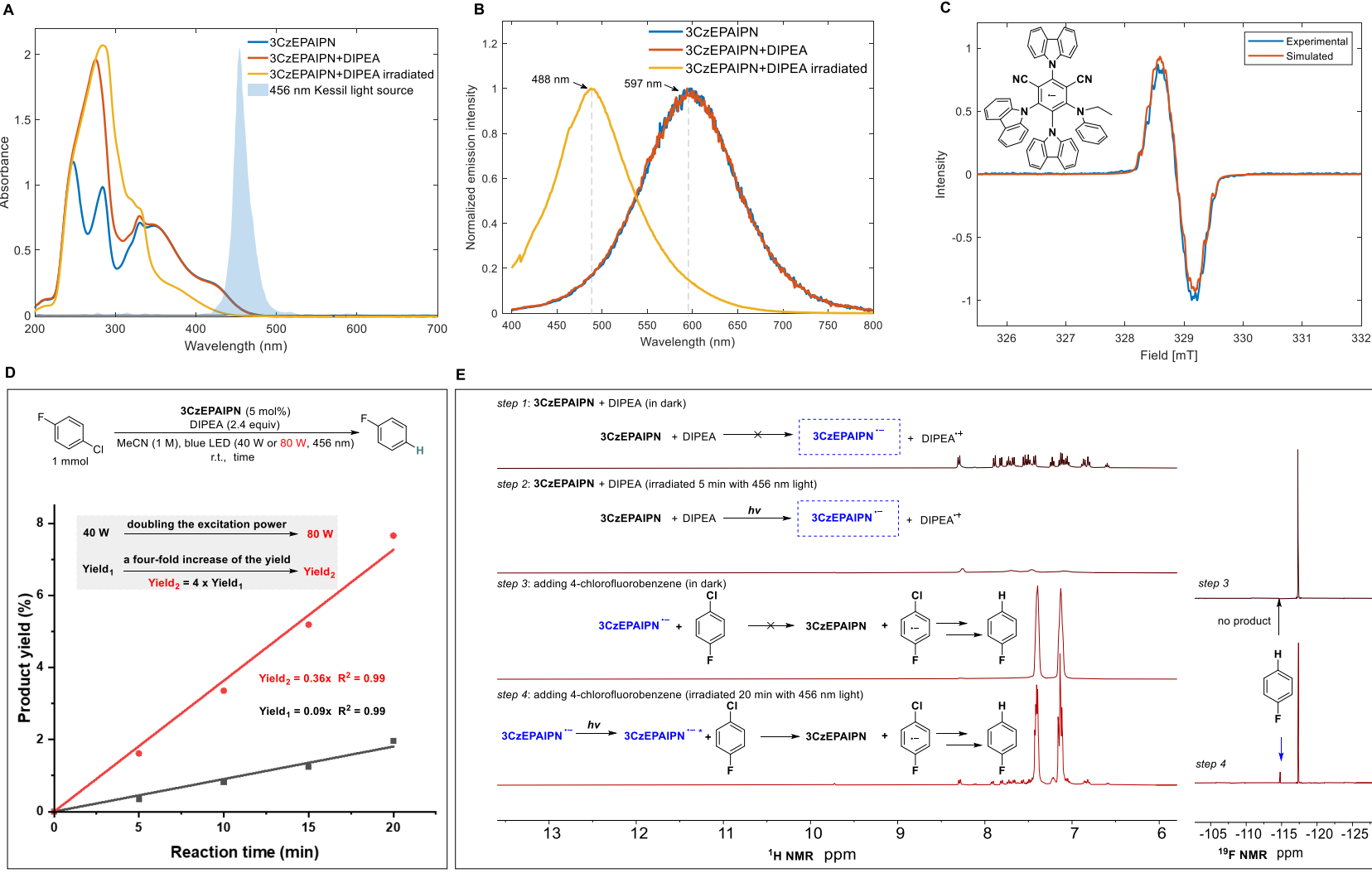

E
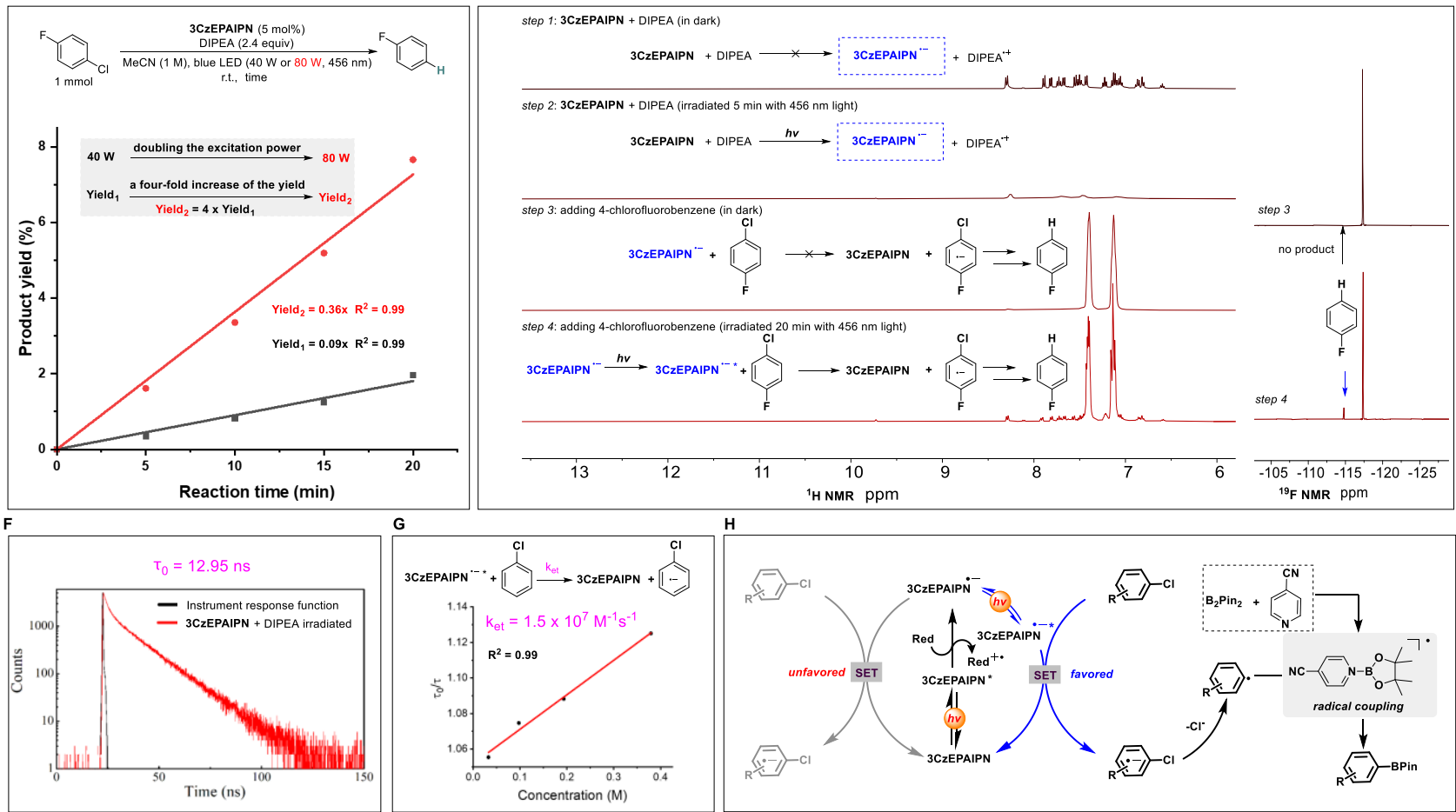

Figure 3. Proposed mechanism with supporting evidence. (A) Absorbance profiles for 3 CzEPAIPN and 3 CzEPAIPN ${ }^{--}\left(3.3 \times 10^{-5} \mathrm{M}, 1.0\right.$ 
Experimental and simulated EPR spectra to support the presence of 3CzEPAIPN ${ }^{--}$. (D) Quadratic dependency of the dechlorination product yield on the irradiation density to support the overall two-photon absorption process. $\mathrm{R}^{2}$ is the coefficient of determination, known as the goodness of fit. (E) NMR spectroscopic evidence supporting the proposed ConPET process. (F) Determination of the

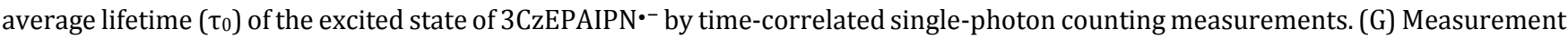

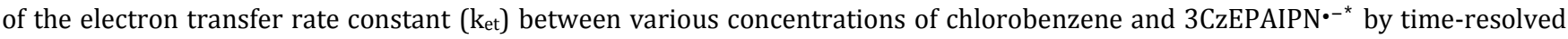
emission quenching experiments. $\tau$ is the lifetime of $3 \mathrm{CzEPAIPN}^{\bullet^{-*}}$ in different concentrations of chlorobenzene. (H) Plausible mechanism of borylation of aryl chlorides through the ConPET process.

agents. Arylphosphonium salts are widely used as organocatalysts, phase transfer reagents, ionic liquids, etc. In addition, they can also be used as functional handles for further C-O, C-S, $\mathrm{C}-\mathrm{N}$, and $\mathrm{C}-\mathrm{C}$ bond formation. ${ }^{37}$ However, existing methods for the preparation of arylphosphonium salts from aryl halides or pseudohalides rely on transition metal catalysis and high-temperature processes. ${ }^{38}$ We herein developed a metal-free synthesis of arylphosphonium salts under ambient conditions using 3CzEPAIPN as the photocatalyst and substoichiometric DIPEA as the electron donor (Figure 2A). Notably, this reactivity enabled engagement of both electron-rich and electronpoor aryl chlorides (42-51), as well as various arylphosphines (52-54), delivering the desired phosphonium salts in good to excellent yields. Moreover, exposure of aryl exclusively in chlorides with trimethyl phosphite to slightly modified conditions enabled a visible light promoted Arbuzov reaction for the generation of aryl phosphonates (55-60, Figure 2B). ${ }^{39}$

The synthetic utility of this ConPET protocol was further extended to dearomative hydroarylation of aryl chlorides to produce spirocyclic cyclohexadienes (61-63), probably through a reductive radical-polar crossover mechanism, as reported by Jui ${ }^{40}$ (Figure 2C). The catalytic system could also be applied to reductive defluorination of trifluoromethylarenes to methylarenes (64, Figure 2D). ${ }^{41}$ Moreover, the developed phosphorylation was amenable to scale-up with an operationally simple continuous-flow setup, which resulted in phosphonium salts in good yield and gram scale production with reduced amounts of $\mathrm{Ph}_{3} \mathrm{P}$ (1.2 equiv) and photocatalyst (2.5 mol\%), highlighting the excellent efficiency of microtubing flow reactors in photochemical synthesis (Figure 2E).

To gain more insight into the proposed ConPET process, various spectroscopic studies and control experiments were performed (Figure 3). First, upon irradiation of a solution of 3CzEPAIPN and DIPEA with a $456 \mathrm{~nm}$ LED, a color change from yellow to brown was observed. UV-visible (UV-Vis) spectroscopy demonstrated a redshift of the maximum absorption peak (Figure 3A). The emission of these mixtures clearly underwent a hypsochromic shift ( $\lambda_{\max }^{\text {em }}$ from 597 to $488 \mathrm{~nm}$, Figure 3B) resulted from the formation of a new species, which is similar to the result observed for 4CzIPN. To verify that this new species was the long-lived radical 3CzEPAIPN ${ }^{--}$, electron paramagnetic resonance (EPR) spectroscopy was conducted. The experimental EPR spectrum matches well with the simulated spectrum of 3CzEPAIPN ${ }^{--}$(Figure 3C). ${ }^{42}$ The quadratic dependency of dechlorination reaction yield on the irradiation intensity further supported that the aryl radical formation mechanism involves an overall two photon excitation (Figure 3D). ${ }^{43}$

Direct evidence of the ConPET process was obtained by ${ }^{1} \mathrm{H}$ NMR investigation (Figure 3E). The characteristic NMR signals of 3CzEPAIPN were detected with a mixture of the photocatalyst and DIPEA in $\mathrm{CD}_{3} \mathrm{CN}$ (step 1). Upon directly exposing the solutions within the NMR tube to blue light, line broadening and signal disappearance were observed, probably due to the generation of unpaired electron species (step 2), which have a strong influence on NMR resonance transverse relaxation rates of neighboring protons, indicating the generation of 3 CzEPAIPN ${ }^{\bullet-}$. This radical anion species was stable under argon for at least $3 \mathrm{~h}$, and could be quenched by air to convert back to the ground state catalyst (Fig. S14). The addition of aryl chlorides showed no effect on the radical anion species in the absence of light (step 3). However, in the presence of light, they quenched the radical anion species back to 3CzEPAIPN, and the dehalogenation product was detected by ${ }^{19} \mathrm{~F}$ NMR spectra, which is consistent with our proposal that photoexcited

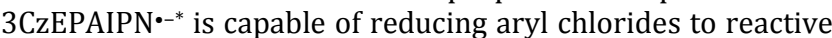
aryl radicals.

The long lifetime of unexcited 3CzEPAIPN ${ }^{*-}$ allows it to absorb a photon using an LED light source to generate the excited state, whose properties were explored by time-correlated single photon counting (TCSPC) (Figure 3F). Excitation of $3 \mathrm{CzEPAIPN}^{*-}$ by irradiation at $375 \mathrm{~nm}$ resulted in a characteristic emission band of 3CzEPAIPN ${ }^{* *}\left(\lambda_{\max }^{e m}=488 \mathrm{~nm}\right)$, whose average lifetime was measured to be $12.95 \mathrm{~ns}$, much longer than the 6.33 ns of 4 CzIPN ${ }^{-*}$. The time-resolved emission quenching experiments revealed a linear photoluminescence lifetime quenching of $3 \mathrm{CzEPAIPN}^{-* *}$ by aryl chlorides, which further supported the ConPET process (Figure 3G).

In light of all the experimental data, a plausible mechanistic pathway was proposed using aryl borylation as a representative example (Figure 3H). Following light excitation, 3CzEPAIPN engages in single-electron transfer with an electron donor to generate a long-lived radical anion species, which is then excited by visible light irradiation again to afford excited $3 \mathrm{CzEPAIPN}^{*-*}$. 3CzEPAIPN ${ }^{-*}$ possesses strong reductive potential to undergo electron transfer with reductively recalcitrant aryl chloride, which generates a reactive aryl radical after fragmentation and reforms $3 \mathrm{CzEPAIPN}$ in the ground state. The resulting aryl radical then couples with a Lewis base stabilized boryl radical, ${ }^{35}$ yielding the desired arylboronate product (see SI for the proposed mechanisms for C-P and C-C bond formations).

In conclusion, we have disclosed that donor-acceptor cyanoarene-based fluorophores can undergo ConPET to access catalysts with exceedingly high reducing power, which complements the well-established single-photon-promoted redox chemistry associated with this family of fluorophores. A new catalyst 3CzEPAIPN can be activated through this strategy to enable robust and efficient activation of aryl chlorides with very negative reduction potentials for $\mathrm{C}-\mathrm{B}, \mathrm{C}-\mathrm{P}$, and $\mathrm{C}-\mathrm{C}$ bond 
formations to furnish a broad scope of arylboronates, arylphosphonium salts, arylphosphonates, and spirocyclic cyclohexadienes. The ConPET process has been unambiguously supported by various spectroscopic investigations. The advantages associated with cyanoarene-based fluorophores such as being inexpensive, metal-free, and modular, allow wide applications in the synthetic community and provide a platform to further explore and understand the design principles for more potent photocatalysts based on the ConPET process.

\section{ASSOCIATED CONTENT}

\section{Supporting Information}

The Supporting Information is available free of charge on the ACS Publications website. DOI:

General procedures, tables of reaction optimizations, analytical $\begin{array}{llll}\text { data, } & \text { and } & \text { spectra }\end{array}$

X-ray data for catalyst 3CzEPAIPN (CIF)

\section{AUTHOR INFORMATION}

\section{Corresponding Authors}

Rong Zhou - College of Chemistry and Chemical Engineering, Taiyuan University of Technology, Taiyuan, 030024, P. R. China; orcid.org/0000-0002-0322-9199; Email: zhourong@tyut.edu.cn

Jie Wu - Department of Chemistry, National University of Singapore, 117545, Singapore; orcid.org/0000-0002- 9865-180X; Email: chmjie@nus.edu.sg

\section{Authors}

Jinhui Xu - Department of Chemistry, National University of Singapore, 117545, Singapore

Jilei Cao - College of Chemistry and Chemical Engineering, Taiyuan University of Technology, Taiyuan, 030024, P. R. China Xiangyang Wu - Division of Chemistry and Biological Chemistry, School of Physical and Mathematical Sciences, Nanyang Technological University, 637371, Singapore

Han Wang - Department of Chemistry, National University of Singapore, 117545, Singapore

Xiaona Yang - College of Chemistry and Chemical Engineering, Taiyuan University of Technology, Taiyuan, 030024, P. R. China Xinxin Tang - Department of Chemistry, National University of Singapore, 117545, Singapore

Ren Wei Toh - Department of Chemistry, National University of Singapore, 117545, Singapore

Edwin K. L. Yeow - Division of Chemistry and Biological Chemistry, School of Physical and Mathematical Sciences, Nanyang Technological University, 637371, Singapore; orcid.org/00000003-0290-4882

\section{Notes}

The authors declare no competing financial interests.

\section{ACKNOWLEDGMENTS}

We are grateful for the financial support provided by Pharma Innovation Programme Singapore (A*STAR, SERC A19B3a0014), the National University of Singapore (R-143000-B60-114), and the National Natural Science Foundation of China (Grant Nos. 22071170 and 21502135). The authors would like to thank Saif A. Khan (NUS Singapore), Shunsuke Chiba (NTU Singapore), Joel M. Hawkins (Pfizer), Thomas Knauber (Pfizer), François Le Lévesque (Merck), Lee Edwards (GSK), and Jean-Philippe Krieger (Syngenta) for helpful discussion.

\section{REFERENCE}

${ }^{1}$ Scott, D. E.; Coyne, A. G.; Hudson, S. A.; Abell, C. Fragment-Based Approaches in Drug Discovery and Chemical Biology. Biochemistry 2012, 51, 4990-5003.

2 Blakemore, D. C.; Castro, L.; Churcher, I.; Rees, D. C.; Thomas, A. W.; Wilson, D. M.; Wood, A. Organic Synthesis Provides Opportunities to Transform Drug Discovery. Nat. Chem. 2018, 10, 383-394.

${ }^{3}$ Marzo, L.; Pagire, S. K.; Reiser, O.; König, B. Visible-Light Photocatalysis: Does It Make a Difference in Organic Synthesis? Angew. Chem. Int. Ed. 2018, 57, 10034-10072.

${ }^{4}$ Romero, N. A.; Nicewicz, D. A. Organic Photoredox Catalysis. Chem. Rev. 2016, 116, 10075-10166.

5 Prier, C. K.; Rankic, D. A.; MacMillan, D. W. C. Visible Light Photoredox Catalysis with Transition Metal Complexes: Applications in Organic Synthesis. Chem. Rev. 2013, 113, 5322-5363.

${ }^{6}$ Tucker, J. W.; Stephenson, C. R. J. Shining Light on Photoredox Catalysis: Theory and Synthetic Applications. J. Org. Chem 2012, 77, 1617-1622.

${ }^{7}$ Cybularczyk-Cecotka, M.; Szczepanik, J.; Giedyk, M. Photocatalytic Strategies for the Activation of Organic Chlorides. Nat. Catal. 2020, 3, 872-886.

${ }^{8}$ Internet Bond-energy Databank (pKa and BDE)--iBonD Home Page. http://ibond.chem.tsinghua.edu.cn or http://ibond.nankai.edu.cn. ${ }_{9}^{9}$ Jiang, M.; Li, H.; Yang, H.; Fu, H. Room-Temperature Arylation of Thiols: Breakthrough with Aryl Chlorides. Angew. Chem. Int. Ed. 2017, 56, 874-879.

${ }^{10}$ Qiao, Y.; Yang, Q.; Schelter, E. J. Photoinduced Miyaura Borylation by a Rare-Earth-Metal Photoreductant: The Hexachlorocerate(III) Anion. Angew. Chem. Int. Ed. 2018, 57, 10999-11003.

${ }^{11}$ Uyeda, C.; Tan, Y.; Fu, G. C.; Peters, J. C. A New Family of

Nucleophiles for Photoinduced, Copper-Catalyzed Cross-Couplings via Single-Electron Transfer: Reactions of Thiols with Aryl Halides Under Mild Conditions $\left(0^{\circ} \mathrm{C}\right)$. J. Am. Chem. Soc. 2013, 135, 9548-9552.

${ }^{12}$ Glaser, F.; Kerzig, C.; Wenger, O. S. Multi-Photon Excitation in Photoredox Catalysis: Concepts, Applications, Methods. Angew. Chem. Int. Ed. 2020, 59, 10266-10284.

${ }^{13}$ Liu, J.; Lu, L.; Wood, D.; Lin, S. New Redox Strategies in Organic Synthesis by Means of Electrochemistry and Photochemistry. ACS Cent. Sci. 2020, 6, 1317-1340.

${ }^{14}$ Ravetz, B. D.; Tay, N. E. S.; Joe, C. L.; Sezen-Edmonds, M.; Schmidt, M. A.; Tan, Y.; Janey, J. M.; Eastgate, M. D.; Rovis, T. Development of a Platform for Near-Infrared Photoredox Catalysis. ACS Cent. Sci. 2020, 6, 2053-2059.

${ }^{15}$ Naumann, R.; Goez, M. First Micelle-Free Photoredox Catalytic Access to Hydrated Electrons for Syntheses and Remediations with a Visible LED or even Sunlight. Chem. - Eur. J. 2018, 24, 17557-17567. 16 Ghosh, I.; Shaikh, R. S.; König, B. Sensitization-Initiated Electron Transfer for Photoredox Catalysis. Angew. Chem. Int. Ed. 2017, 56, 8544-8549.

${ }^{17}$ Naumann, R.; Kerzig, C.; Goez, M. Laboratory-scale Photoredox Catalysis Using Hydrated Electrons Sustainably Generated with a Single Green Laser. Chem. Sci. 2017, 8, 7510-7520.

${ }^{18}$ Kim, H.; Kim, H.; Lambert, T. H.; Lin, S. Reductive

Electrophotocatalysis: Merging Electricity and Light to Achieve Extreme Reduction Potentials. J. Am. Chem. Soc. 2020, 142, 20872092.

${ }^{19}$ Cowper, N. G. W.; Chernowsky, C. P.; Williams, O. P.; Wickens, Z. K. Potent Reductants via Electron-Primed Photoredox Catalysis: Unlocking Aryl Chlorides for Radical Coupling. J. Am. Chem. Soc. 2020, 142, 2093-2099.

${ }^{20}$ Targos, K.; Williams, O. P.; Wickens, Z. K. Unveiling Potent Photooxidation Behavior of Catalytic Photoreductants. J. Am. Chem. Soc. 2021, 143, 4125-4132. 
${ }^{21}$ Ghosh, I.; Ghosh, T.; Bardagi, J. I.; Konig, B. Reduction of Aryl Halides by Consecutive Visible Light-Induced Electron Transfer Processes. Science 2014, 346, 725-728.

22 Ghosh, I.; König, B. Chromoselective Photocatalysis: Controlled Bond Activation through Light-Color Regulation of Redox Potentials. Angew. Chem. Int. Ed. 2016, 55, 7676-7679.

${ }^{23}$ Neumeier, M.; Sampedro, D.; Májek, M.; de la Peña O'Shea, V. A.; Jacobi von Wangelin, A.; Pérez-Ruiz, R. Dichromatic Photocatalytic Substitutions of Aryl Halides with a Small Organic Dye. Chem. - Eur. J. 2018, 24, 105-108.

${ }^{24}$ Bardagi, J. I.; Ghosh, I.; Schmalzbauer, M.; Ghosh, T.; König, B. Anthraquinones as Photoredox Catalysts for the Reductive Activation of Aryl Halides. Eur. J. Org. Chem. 2018, 2018, 34-40.

${ }^{25}$ MacKenzie, I. A.; Wang, L.; Onuska, N. P. R.; Williams, O. F.; Begam, K.; Moran, A. M.; Dunietz, B. D.; Nicewicz, D. A. Discovery and Characterization of an Acridine Radical Photoreductant. Nature 2020, 580, 76-80.

${ }^{26}$ Cole, J. P.; Chen, D.-F.; Kudisch, M.; Pearson, R. M.; Lim, C.-H.; Miyake, G. M. Organocatalyzed Birch Reduction Driven by Visible Light. J. Am.

Chem. Soc. 2020, 142, 13573-13581.

${ }^{27}$ During the process of our manuscript preparation, König et al. reported an excellent ipso-borylation of substituted arenes by using thiolate as a catalyst, see: Chem 2021, In Press,

doi.org/10.1016/j.chempr.2021.04.016.

${ }^{28}$ Uoyama, H.; Goushi, K.; Shizu, K.; Nomura, H.; Adachi, C. Highly Efficient Organic Light-emitting Diodes from Delayed Fluorescence. Nature 2012, 492, 234-238.

${ }^{29}$ Luo, J.; Zhang, J. Donor-Acceptor Fluorophores for Visible-LightPromoted Organic Synthesis: Photoredox/Ni Dual Catalytic C( $\left.\mathrm{sp}^{3}\right)-$ $\mathrm{C}\left(\mathrm{sp}^{2}\right)$ Cross-Coupling. ACS Catal. 2016, 6, 873-877.

${ }^{30}$ Shang, T.-Y.; Lu, L.-H.; Cao, Z.; Liu, Y.; He, W.-M.; Yu, B. Recent Advances of 1,2,3,5-Tetrakis(carbazol-9-yl)-4,6-dicyanobenzene (4CzIPN) in Photocatalytic Transformations. Chem. Commun. 2019, 55, 5408-5419.

${ }^{31}$ Speckmeier, E.; Fischer, T. G.; Zeitler, K. A Toolbox Approach To Construct Broadly Applicable Metal-Free Catalysts for Photoredox Chemistry: Deliberate Tuning of Redox Potentials and Importance of Halogens in Donor-Acceptor Cyanoarenes. J. Am. Chem. Soc. 2018, 140, 15353-15365.

32 Tian, Y.-M.; Guo, X.-N.; Krummenacher, I.; Wu, Z.; Nitsch, J.; Braunschweig, H.; Radius, U.; Marder, T. B. Visible-Light-Induced NiCatalyzed Radical Borylation of Chloroarenes. J. Am. Chem. Soc. 2020, 142, 18231-18242.

33 Jin, S.; Dang, H. T.; Haug, G. C.; He, R.; Nguyen, V. D.; Nguyen, V. T.; Arman, H. D.; Schanze, K. S.; Larionov, O. V. Visible Light-Induced Borylation of C-O, C-N, and C-X Bonds. J. Am. Chem. Soc. 2020, 142, 1603-1613.

${ }^{34}$ Zhang, L.; Jiao, L. Visible-Light-Induced Organocatalytic Borylation of Aryl Chlorides. J. Am. Chem. Soc. 2019, 141, 9124-9128.

35 Takaoka, A.; Yokokohji, O.; Yamaguchi, Y.; Isono, T.; Motoyoshi, M.; Ishikawa, N. Preparation of Tetrafluoroisophthalonitrile and Its Nucleophilic Substitution at 4-Position. Nippon Kagaku Kaishi 1985, 1985, 2155-2160.

${ }^{36}$ Wang, G.; Zhang, H.; Zhao, J.; Li, W.; Cao, J.; Zhu, C.; Li, S. Homolytic Cleavage of a B-B Bond by the Cooperative Catalysis of Two Lewis Bases: Computational Design and Experimental Verification. Angew. Chem. Int. Ed. 2016, 55, 5985-5989.

${ }^{37}$ Hilton, M. C.; Dolewski, R. D.; McNally, A. Selective Functionalization of Pyridines via Heterocyclic Phosphonium Salts. J. Am. Chem. Soc.

2016, 138, 13806-13809.

${ }^{38}$ Huang, W.; Zhong, C. H. Metal-Free Synthesis of Aryltriphenylphosphonium Bromides by the Reaction of Triphenylphosphine with Aryl Bromides in Refluxing Phenol. ACS Omega 2019, 4, 6690-6696.

${ }^{39}$ Shaikh, R. S.; Düsel, S. J. S.; König, B. Visible-Light Photo-Arbuzov Reaction of Aryl Bromides and Trialkyl Phosphites Yielding Aryl Phosphonates. ACS Catal. 2016, 6, 8410-8414.

${ }^{40}$ Flynn, A. R.; McDaniel, K. A.; Hughes, M. E.; Vogt, D. B.; Jui, N. T. Hydroarylation of Arenes via Reductive Radical-Polar Crossover. J. Am. Chem. Soc. 2020, 142, 9163-9168.
${ }^{41}$ Sap, J. B. I.; Straathof, N. J. W.; Knauber, T.; Meyer, C. F.; Medebielle, M.; Buglioni, L.; Genicot, C.; Trabanco, A. A.; Noel, T.; Am Ende, C. W.; Gouverneur, V. Organophotoredox Hydrodefluorination of Trifluoromethylarenes with Translational Applicability to Drug Discovery. J Am Chem Soc 2020, 142, 9181-9187.

${ }^{42}$ Stoll, S.; Schweiger, A. EasySpin, a comprehensive software package for spectral simulation and analysis in EPR. J. Magn. Reson. 2006, 178, 42-55.

${ }^{43}$ Chatterjee, A.; König, B. Birch-Type Photoreduction of Arenes and Heteroarenes by Sensitized Electron Transfer. Angew. Chem. Int. Ed. 2019, 58, 14289-14294.

\section{For Table of Contents only}

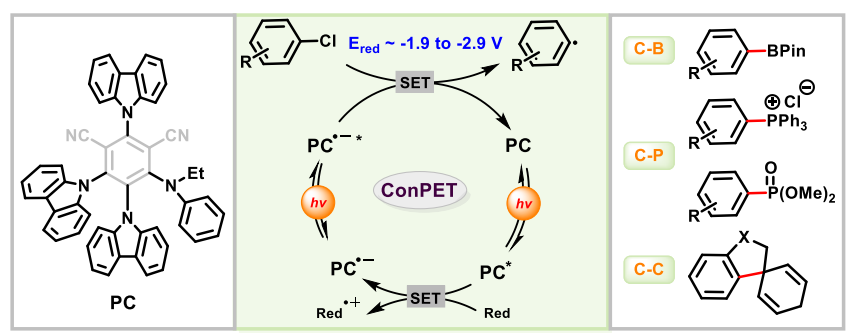

